\title{
Implementation of Robust EPSAC on dynamic walking of COMAN Humanoid
}

\author{
Juan A. Castano* Andres Hernandez ${ }^{* *}$ Zhibin Li ${ }^{*}$ \\ Chengxu Zhou* Nikos G. Tsagarakis* Darwin G. Caldwell* \\ Robin De Keyser** \\ * Department of Advanced Robotics, (Fondazione) Istituto Italiano di \\ Tecnologia, via Morego, 30, 16163 Genova, Italy (Juan.Castano@iit.it) \\ ** Department of Electrical energy, Systems and Automation, Ghent \\ University, Belgium (Andres.Hernandez@UGent.be)
}

\begin{abstract}
In this paper we present the Model Predictive Control (MPC) with dynamic constraints for generating dynamic walking for the compliant humanoid COMAN. The dynamics of the robot are modeled using the cart-table model which allows the generation of a dynamically balanced gait given a planned walking pattern based on the Zero Moment Point (ZMP). Our simulation study of the MPC's implementation on bipedal walking finds out that a large receding and control horizons are needed to track a predefined walking pattern, leading to numerical instability. Therefore, the Extended Prediction Self-Adaptive Control (EPSAC) approach for MPC has been used and a method based on the analysis of the Singular Value Decomposition (SVD) is presented as new contribution to guarantee feasibility, robustness and stability of the MPC formulation. Study on an inverted pendulum and the COMAN humanoid prove that the proposed strategy improves the robustness and stability of the original EPSAC controller, in both well or ill conditioned systems. The simulation results finally demonstrate that the proposed methodology is well suited to smoothly track a dynamic walking pattern.
\end{abstract}

\section{INTRODUCTION}

Biped locomotion has been widely studied in recent decades. The capability of bipedal robots to walk in the environment designed for humans is particularly noteworthy. To realize this, the walking gait should be adapted to the terrain and continuously regulated according to the robot's real state. Thus control techniques are demanded to generate reactive response to the unpredicted terrain, obstacles, and transitions during the gait trajectory. A possible approach to solve this problem is to apply predictive techniques to generate a proper adaptive gait.

A common control approach to generate the dynamically stable walking pattern is based on the Zero Moment Point (ZMP). The ZMP can be interpreted as the point on the ground where all the inertial and gravity forces have no component along the horizontal axes (Vukpbratovic and Boravac [2004]). While the ZMP remains inside the support polygon, the robot will not tip around the stance foot (Vanderborght [2010]). This technique has been studied in different works (Vukobratovic and Juricic [1969],Kajita et al. [2003]), nonetheless, the problem of physical constraints was avoided by place the ZMP always in the center of support polygon.

The preview controller, which is presented in e.g. (Kajita et al. [2003]) has been widely used. In that work the

\footnotetext{
* This work is supported by the FP7 European projects AMARSi (ICT-248311) and WALK-MAN (ICT-2013-10).

Andres Hernandez acknowledge the financial support provided by the Institute for the Promotion and Innovation by Science and Technology in Flanders (IWT SBO-110006).
}

generation of the COM trajectories based on the tracking of ZMP reference is shown. In that work the authors described the ZMP concept as well as the implementation of the preview controller to track a desire trajectory. That strategy applied a fix pattern generator.

In order to achieve the biped locomotion and include constraints in the desired gait, the Model Predictive Control (MPC) is of particular interest, as it has an inherent deadtime compensation, includes feedforward action and deals with constrained systems. In particular, the Extended Prediction Self-Adaptive Control (EPSAC) proposed in (De Keyser [2003]) has been chosen in our work due to its flexibility to model the disturbances. In addition, this technique permits the implementation of input, inputrate and output constraints, which represent the physical constraints in dynamic walking. The long prediction and control horizons particularly required by the bipedal walking has an instability issue due to the inversion of large matrices, hence, it is necessary to include conditions to guarantee the feasibility.

The work in (Parsa and Farrokhi [2010]), presents a Nonlinear predictive control that uses a complex dynamic model for the single and double support phases, additionally a disturbance observer is included in the loop to compensate for external disturbances. Stability or robust analysis of the used method are not included. A linear implementation of the MPC is presented in (Bagheri and Miripour-Fard [2011]), where the authors applied the MPC to control the biped locomotion. Feasibility is guaranteed by imposing fixed input and output constraints, and long 
prediction and control horizons. No further analysis on stability and robustness of the method was presented.

The previous gait control developed for COMAN in ( $\mathrm{Li}$ et al. [2013]) utilized the COM state for online generating walking gait rather than relying on ZMP, however, gait control was not optimal. We further extend the use of COM state feedback for updating the step length and thus the ZMP reference during walking, and apply MPC to smoothly produce a reactive and dynamically

Our work provides a robust extension of constrained linear Model Predictive Control (EPSAC) (De Keyser [2003]) generating a walking pattern of a humanoid robot basedon the ZMP (Vukpbratovic and Boravac [2004]). The implementation of the MPC controller for this issue leads to the conclusion that large prediction and control horizons are required, resulting in numerical instability in the control (De Keyser [2003]). Inspired by (Rojas et al. [2004]), a method based on the Singular Value Decomposition (SVD) is proposed to improve the robustness and stable characteristics of the EPSAC controller for both well- and ill-conditioned systems. An inverted pendulum and the bipedal walking cases are studied to demonstrate the effectiveness of the proposed control algorithms. The method uses the impulse response to provide a well-defined matrix for the EPSAC controller when large control and prediction horizons are required, providing robustness to the whole system.

\section{EPSAC ALGORITHM}

MPC is a general designation for controllers that make an explicit use of a model of the plant to obtain the control signal by minimizing an objective function over a time horizon. In this contribution, EPSAC proposed by (De Keyser [2003]) has been chosen due to its easy implementation of constraints. The EPSAC methodology is described as follows: Having a system

$$
y(t)=f\left(y_{t-1}, y_{t-2}, \ldots, u_{t-1}, u_{t-2}, \ldots\right)
$$

with input $u(t)$ and process output $y(t)$. The EPSAC uses the modeled output

$$
x(t)=f\left(x_{t-1}, x_{t-2}, \ldots, u_{t-1}, u_{t-2}, \ldots\right) .
$$

The generic model of the EPSAC is (1)

$$
y(t)=x(t)+n(t),
$$

where $y(t)$ is the measured output of the process, $x(t)$ the model output and $n(t)$ a process disturbance, all at discrete-time index $t$. The disturbance $n(t)$ can be modeled as colored noise through a filter with the transfer function

$$
n(t)=\frac{C\left(q^{-1}\right)}{D\left(q^{-1}\right)} e(t)
$$

with $e(t)$ uncorrelated (white) noise with zero-mean and $C, D$ monic polynomials in the backward shift operator $q^{-1}$. The disturbance model allows to achieve robustness of the control loop. A 'default' choice to remove steady-state control offsets is $n(t)=\frac{1}{1-q^{-1}} e(t)$ (Maciejowski [2002]). However, a higher performance is achieved by using an 'intelligent' disturbance model (De Keyser and Ionescu [2003]), making it to suit the type of disturbance.

Using the generic process model (1), the predicted values of the output are

$$
y(t+k \mid t)=x(t+k \mid t)+n(t+k \mid t)
$$

for $k=N_{1}, N_{1}+1, \ldots, N_{2} \mid N_{1}, N_{2} \in \Re$, where $N_{1}$ and $N_{2}$ are the minimum and the maximum prediction horizons. The prediction of the process output is based on the measurements available at the sampling time instant $t$, $\{x(t-1), x(t-2), \ldots, u(t-1), u(t-2), \ldots\}$ and future (postulated) values of the input signal $\{u(t \mid t), u(t+1 \mid t), \ldots\}$. The future response can then be expressed as

$$
y(t+k \mid t)=y_{\text {base }}(t+k \mid t)+y_{\text {opt }}(t+k \mid t),
$$

where each of the contribution terms is understood as:

- $y_{\text {base }}(t+k \mid t)$ is the effect of the past inputs $u(t-$ 1), $u(t-2) \ldots$, a future base control sequence $u_{\text {base }}(t+$ $k \mid t)$ that can be the last used input and the predicted disturbance $n(t+k \mid t)$.

- $y_{\text {opt }}(t+k \mid t)$ is the effect of the optimizing control actions $\delta u(t \mid t), \ldots, \delta u\left(t+N_{u}-1 \mid t\right)$ with $\delta u(t+k \mid t)=$ $u(t+k \mid t)-u_{\text {base }}(t+k \mid t)$, in a control horizon $N_{u}$.

The optimized output $y_{\text {opt }}(k) \forall k=\left[1,2, \ldots, N_{2}\right]$ can be expressed as the discrete time convolution of the unit impulse response coefficients $h_{1}, \ldots, h_{N_{2}}$ and unit step response coefficients $g_{1}, \ldots, g_{N_{2}}$ of the system.

$$
\begin{array}{r}
y_{\text {opt }}(t+k \mid t)=h_{k} \delta u(t \mid t)+h_{k-1} \delta u(t+1 \mid t)+\ldots \\
+g_{k-N_{u}+1} \delta u\left(t+N_{u}-1 \mid t\right)
\end{array}
$$

Using (3) and (4), the key EPSAC formulation becomes

$$
\mathbf{Y}=\overline{\mathbf{Y}}+\mathbf{G} \mathbf{U}
$$

where

$$
\begin{aligned}
& \mathbf{Y}=\left[y\left(t+N_{1} \mid t\right) \ldots y\left(t+N_{2} \mid t\right)\right]^{T} \\
& \overline{\mathbf{Y}}=\left[y_{\text {base }}\left(t+N_{1} \mid t\right) \ldots y_{\text {base }}\left(t+N_{2} \mid t\right)\right]^{T} \\
& \mathbf{U}=\left[\delta u(t \mid t) \ldots \delta u\left(t+N_{u}-1 \mid t\right)\right]^{T} \\
& \mathbf{G}=\left[\begin{array}{cccc}
h_{N_{1}} & h_{N_{1}-1} \ldots & g_{N_{1}-N_{u}+1} \\
h_{N_{1}+1} & h_{N_{1}} & \ldots & \ldots \\
\ldots & \ldots & \ldots & \ldots \\
h_{N_{2}} & h_{N_{2}-1} & \ldots & g_{N_{2}-N_{u}+1}
\end{array}\right]
\end{aligned}
$$

Then, the control signal $\mathrm{U}$ is optimized by minimizing the cost function:

$$
\sum_{k=N_{1}}^{N_{2}}[r(t+k \mid t)-y(t+k \mid t)]^{2}
$$

Note that the controller cost function (7) can be easily extended to many alternative cost functions as described in (De Keyser [2003]). The horizons $N_{1}, N_{2}$ and $N_{u}$ are design parameters and

$$
r(t+k \mid t)=\alpha r(t+k-1 \mid t)+(1-\alpha) w(t+k \mid t)
$$

is the desired reference trajectory, where a $1^{\text {st }}$-order trajectory was chosen for $k=1, \ldots, N_{2}$ with initialization $r(t \mid t)=y(t)$. The signal $w(t)$ represents the setpoint and alpha $(\alpha)$ is a design parameter to tune the MPC performance (Sánchez and Rodellar [1996]).

The cost function (7) can be represented in its compact matrix notation as follows:

$$
(\mathbf{R}-\mathbf{Y})^{\mathbf{T}}(\mathbf{R}-\mathbf{Y})=[(\mathbf{R}-\overline{\mathbf{Y}})-\mathbf{G} \mathbf{U}]^{\mathbf{T}}[(\mathbf{R}-\overline{\mathbf{Y}})-\mathbf{G U}]
$$

where $\mathbf{R}=\left[r\left(t+N_{1} \mid t\right) \ldots r\left(t+N_{2} \mid t\right)\right]^{T} \in \Re^{N_{2}}$. The previous expression can be easily transformed into the standard quadratic cost index:

$$
J(\mathbf{U})=\mathbf{U}^{\mathbf{T}} \mathbf{H} \mathbf{U}+\mathbf{2} \mathbf{f} \mathbf{U}+c .
$$


with,

$$
\begin{array}{r}
\mathbf{H}=\mathbf{G}^{\mathbf{T}} \mathbf{G} \quad \mathbf{f}=-\mathbf{G}^{\mathbf{T}}(\mathbf{R}-\overline{\mathbf{Y}}) \\
c=(\mathbf{R}-\overline{\mathbf{Y}})^{\mathbf{T}}(\mathbf{R}-\overline{\mathbf{Y}})
\end{array}
$$

where $\left[G^{T} G\right] \in \Re^{N_{u} \times N_{u}}$. In practice, all processes are subject to constraints. For the case of limits in the actuators range (input constraints), two approaches are available:

Clipping is the simplest approach as the control is calculated assuming the actuator has unlimited range, for which an analytical solution is available:

$$
\mathbf{U}^{*}=\left[\mathbf{G}^{\mathbf{T}} \mathbf{G}\right]^{-\mathbf{1}}\left[\mathbf{G}^{\mathbf{T}}(\mathbf{R}-\overline{\mathbf{Y}})\right]
$$

Once the action $U^{*}$ is calculated, it is then hard-limited into a minimum and maximum allowed value.

Constrained control, constraints are taken into account a priori, thus leading to the best solution that is possible within the specified limits. In MPC, the calculation of these constraints is the so called quadratic programming optimization problem.

Finally, the feedback characteristic of MPC is given by the first optimal control input $u^{*}(t)=u_{\text {base }}(t \mid t)+\delta u(t \mid t)=$ $u_{\text {base }}(t \mid t)+U^{*}(1)$ is applied to the plant and then the whole procedure is repeated at the next sampling time $t+1$.

\section{ROBUST EXTENSION FOR THE EPSAC BASED ON SVD}

As cited in (Rojas and Goodwin [2004],Rojas et al. [2004]), the stability information of the system can be extracted from the Singular Value Decomposition (SVD). In the EPSAC methodology, the computation of the optimal control input $\delta u(t)$ implies the inversion of the $G$, consequently, numerical stability strongly depends on whether or not this matrix is well-defined. $G$ is composed of $\left(N_{u}\right)$ singular values arranged in a decreasing continuous function. Herein, we present a method based on the impulse response in order to provide a well-defined matrix $G$ for large control and prediction horizons $N_{u}$ and $N_{2}$ based on the SVD, providing robustness to the whole system.

Given (6), with $N_{1}=1$ and knowing that $h_{k}=0, g_{k}=$ $0 \forall k<0 \mid k \in Z$ then, $G$ is a bottom triangular matrix. The SVD representation of $\mathrm{G}$ is

$$
G=T \Sigma V^{T}
$$

where $T=\left[T_{1}, T_{2}, \ldots, T_{N_{2}}\right] \in \Re^{N_{2} \times N_{u}}$ represents the left singular vectors, $V=\left[v_{1}, v_{2}, \ldots, v_{N_{2}}\right] \in \Re^{N_{u} \times N_{2}}$ the right singular vectors, and $\Sigma=\operatorname{diag}\left[\sigma_{1}, \sigma_{2}, \ldots, \sigma_{N_{u}}\right] \in \Re^{N_{u} \times N_{u}}$ the singular values of $\mathrm{G}$. From a geometrical point of view, the SVD creates a rotated hypersphere $\in \Re_{u}^{N}$ that belongs to the space $\Re^{N_{2}}$ and the corresponding $\sigma_{i} i \in$ $\left[1,2, \ldots, N_{u}\right]$ value, defines the length of each direction.

It is desired not to reduce the solution space; then, a modification of the hyperspace is proposed. The method changes only the magnitude of the singular values $\sigma_{i} \forall i \in$ $K \mid K \leq N_{u}$, while the singular vectors remain constant. So, a bigger hypersphere containing the nominal solution space is generated. The proposed method allows a softer transition and higher correlation between the $N_{u}$ spaces. Taking into account the response of the system through the time and adapting the singular values to increase the correlation of the input in the control horizon $N_{u}$, we obtain a response that is robust against uncertainties in the model. The proposed method allows to consider the complete control horizon $N_{u}$ during the optimization phase since the singular values $\sigma$ are disperse according to the impulse response of the system. For this aim, a close relation between the value $\sigma_{j} \mid j \in Z \leq N_{u}$ and the response of the system at time $t+j$ is defined.

\subsection{Hypersphere construction}

Consider a system with parameters $L=\left[l_{1}+\delta_{1}, l_{2}+\right.$ $\left.\delta_{2}, \ldots, l_{m}+\delta_{m}\right]$ and impulse response $H_{L}$, where $\delta_{i}$ is the variation of the $i^{\text {th }}$ parameter, and let $G$ be as in (6), then a SVD representation of the system is obtained with $\Sigma_{L}=\left[\sigma_{L_{L} \delta_{1}}, \sigma_{2_{L} \delta_{2}}, \ldots, \sigma_{N u_{L} \delta_{m}},\right]$. Since $\Sigma_{L}$ is affected by the parametrization of the model, each SVD $\sigma_{i_{L} \delta_{i}}$ is affected as well. This representation maps the effect of the parameter $l_{i}+\delta_{i}$ into the SVD of the system providing a solution space to be controlled. In order to build a robust representation of matrix $G$ based on its SVD, it is possible to take the maximum $\sigma_{i_{L} \delta_{i}}$ for each row in $\Sigma_{L}$. Nevertheless, we propose a fitting function based on the impulse response of the nominal system that provides a soft transition though the hyper spaces of $\Sigma_{L}$, increasing the relation of the optimal input $U_{t}$, to be applied to the system, with respect to the control horizon inputs $U=\left[U_{t}, U_{t+1}, \ldots, U_{t+N_{u}}\right]$.

To generate the hypersphere, note that the $i^{\text {th }}$ row of $G$ contains the impulse response for $1 \leq j \leq N_{u}-1$, where $G(i, j)=0 \forall j>i$. Then, the percentage magnitudes of the contribution $C_{p}(k)$ of $h_{N 1}$ applied at future time $K_{f}=\left[1,2, \ldots, N_{u}\right]$ are;

$$
C_{p}(k)=\frac{\left|h_{N 1} 100 \%\right|}{\sum_{j=1}^{k}|G(k, j)|}, \forall k \leq N_{u},
$$

The matrix $\Sigma_{C}$ is based on $\Sigma, \Sigma_{C_{1}}=\sigma_{1}$, and $\Sigma_{C_{N_{u}}}=\sigma_{N_{u}}$. Then, $C_{p}(1)=100 \%$ is referred to $\Sigma_{C_{1}}$. The remaining $C_{p}$ values correspond to the other values of the diagonal matrix $\Sigma_{C}$ keeping the proportion provided by the impulse responses relation.

\subsection{Robust Analysis}

In order to understand the effect of $\Sigma$ in $G$, first consider $G \in \Re^{N_{2} \times N_{u}}$ which implies that the expected optimization that provides the control effort at present time $t$ is obtained by a minimization process affected by the future inputs up to time $t+N_{u}$. From the SVD, we have that the effect of the input at time $t+N_{i}, \forall 0<N_{i} \leq N_{u}$ is directly related with the corresponding value $\sigma_{N i}$ in $\Sigma$. In order to guarantee a robust response of the system given the uncertainties $\delta$, the hyperspace described by $\Sigma_{C}$ must contain the complete space solution of the system $L$. The proposed method constructs the hyper space using the maximum $\sigma$ value at the first row of $\Sigma_{L^{\delta}}$ and using (12), the new SVD is presented containing the hyper space solution. The approximation provides a wider hyper sphere that contains the original space solution using the impulse response contributions in $G$. Because of the sparse modification on $G$ while the prediction model remains constant, the constraints may not be completely respected. So, soft constraints should be implemented to guarantee a solution 
in the optimization. Other solution to this problem is given in (Wang [2010]), where the cost function is modified using weights on the control horizon to reduce the effect introduced by long prediction horizon.

For well defined problems, the method slightly affects the original SVD representation as shown in Section 3.3, since the new SVD representation is close to the original one and the right and left singular vectors are not changed. I.e. the original space and the approximated solution are equivalent. To better illustrate this concept, the inverted pendulum is used as an example.

\subsection{Validation on an inverted pendulum}

The inverted pendulum is a nonlinear and unstable system which has non-minimum phase behavior. In this example, we consider a two-dimensional inverted pendulum with the cart constrained to move in the horizontal plane, as depicted in Fig. 1. The control input is the horizontal force $F$, and the outputs are the angular position of the pendulum $\theta$ and the horizontal position of the cart $x$. The system nonlinear differential equations are:

$$
\begin{aligned}
& (M+m) \ddot{x}+b \dot{x}+m l \ddot{\theta} \cos \theta-m l \dot{\theta}^{2} \sin \theta=F \\
& \left(I+m l^{2}\right) \ddot{\theta}+m g l \sin \theta=-m l \ddot{x} \cos \theta
\end{aligned}
$$

The system (13) is linearized at $\theta=\pi$ with the parameters listed on table 1 . The inverted pendulum is a well-

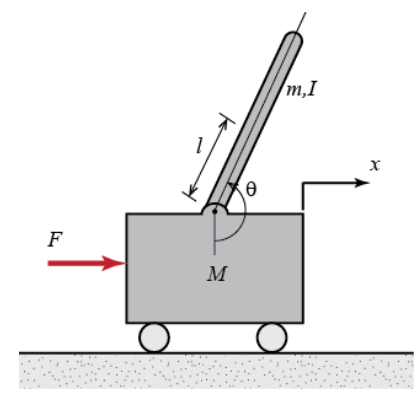

Fig. 1. Schematic representation of the Inverted Pendulum.

Table 1. Parameters for the inverted pendulum

\begin{tabular}{|l|c|r|}
\hline Symbol & Definition & value \\
\hline$M$ & Mass of the car & $0.5 \mathrm{Kg}$ \\
$m$ & mass of the pendulum & $0.2 \mathrm{Kg}$ \\
$b$ & coefficient of friction for cart & $0.1 \mathrm{~N} / \mathrm{m} / \mathrm{sec}$ \\
$l$ & length to pendulum center of mass & $0.3 \mathrm{~m}$ \\
$I$ & moment of inertia of the pendulum & $0.006 \mathrm{~kg} \cdot \mathrm{m}^{2}$ \\
$F$ & force applied to the cart & $\mathrm{N}$ \\
$x$ & cart position coordinate & $\mathrm{m}$ \\
$\theta$ & pendulum angle from vertical (down) & $\mathrm{rad}$ \\
\hline
\end{tabular}

conditioned problem for which a good closed performance can be achieved with a prediction horizon $N_{2}=10$ and control horizon $N_{u}=1$ as depicted in Fig. 2. During the simulations, the EPSAC controller is subject to input and slew-rate constraints defined as: $-20 N \leq u(t+$ $k \mid t) \leq 20 \mathrm{~N}$ and $-0.5 \mathrm{~N} / \mathrm{ms} \leq \Delta u(t+k \mid t) \leq 0 . \overline{5} \mathrm{~N} / \mathrm{ms}$, respectively and a sample time $T_{s}=10 \mathrm{~ms}$.

Assume that a faster response is required, then, two options are available: to decrease the prediction horizon $N_{2}$ or to increase the control horizon $N_{u}$. The latter is less desired since it decreases the robustness and stability of the control loop. To illustrate this behavior, the EPSAC controller was tuned for a larger control horizon $N_{u}=5$, leading to a faster response of the system, generating an undesired oscillation in the control effort. An improvement on the performance of the controller in terms of robustness and stability is achieved by implementing the proposed Robust EPSAC strategy as shown in Fig. 2. The singular
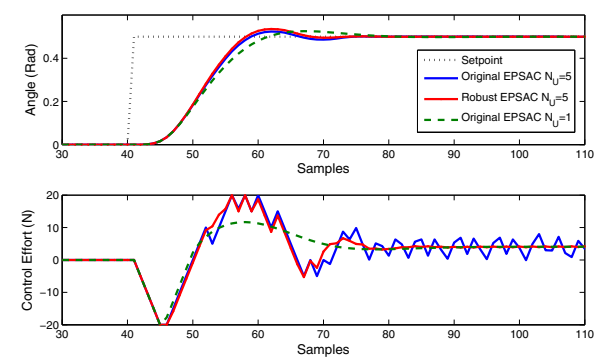

Fig. 2. Comparison of Robust EPSAC for the inverted pendulum system for large control horizon $N_{u}$.

values for the solutions with control horizon $N_{u}=5$ are shown in Fig. 3. The singular values for the Robust EPSAC are close to the original SVD, but far from the singularity compared to the original EPSAC, leading to a smoother and numerically more stable computation of the optimal control action. Furthermore, a robustness test is performed

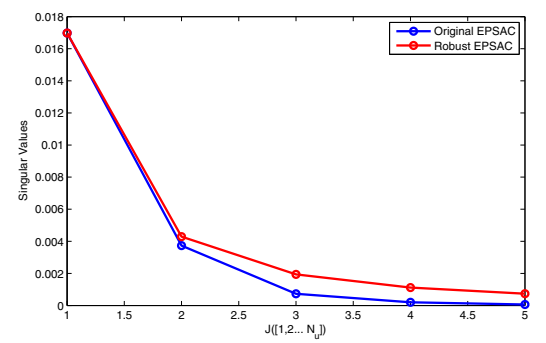

Fig. 3. SVD for the Inverted Pendulum $N_{2}=10, N_{u}=5$.

by changing the masses $M=0.9 \mathrm{Kg}$ and $m=0.6 \mathrm{Kg}$, and the length of the pendulum $l=0.4 \mathrm{~m}$ on the nonlinear model, while the linear model used for prediction remains as listed on table 1. As observed in Fig. 4 the proposed method is more robust compared to the original EPSAC, producing less oscillations and therefore a faster response.
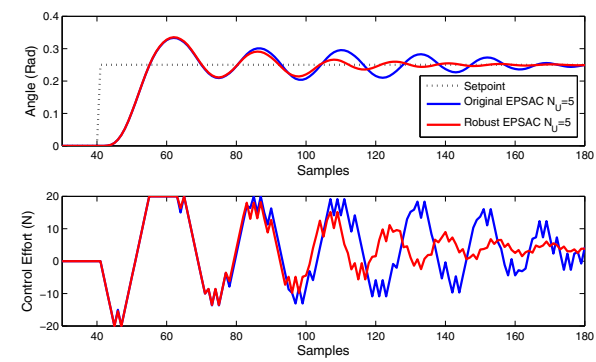

Fig. 4. Robustness Test for the inverted pendulum.

\section{DYNAMIC WALKING OF COMAN HUMANOID}

The car-table model proposed by (Kajita et al. [2003]) was chosen for representing the dynamics of bipedal walking. 


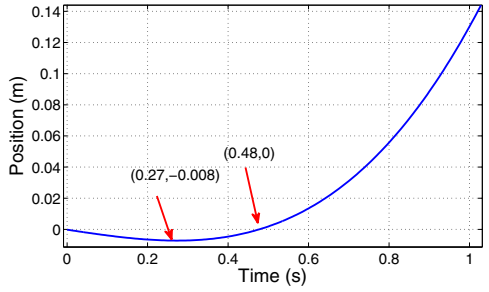

Fig. 5. Cart table model step response. $z c=0.41 \mathrm{~m}$.

The humanoid walking robot was simplified as a cart on top of a mass-less table. The cart represents the center of mass of the robot while the table represents the supporting foot. Given the cart's acceleration, the ZMP position can be computed by

$$
x_{Z M P}=x-\frac{z_{c} \ddot{x}}{g},
$$

where $g$ is the gravity constant and $z_{c}$ is constant the height of the Center of Mass (COM). The output of the system is the ZMP and the control input is the Jerk, defined as the rate of change of acceleration.

The walking characteristics is briefly described to clarify the generation of the ZMP trajectory and the corresponding motion of the COM. The gait is divided as (Edmond Ayyappa [1997]), by the Double Support (DS) and Single Support (SS) phases. The DS phase is the portion of the gait cycle when both feet are in contact with the floor and occupies $25 \%$ of the gait cycle, while the SS phase occupies $75 \%$ of the cycle. During the SS phase, the COM changes while the ZMP remains in the support foot. During the DS phase the ZMP evolves from one foot to the other.

Consider a given $Z M P$ trajectory represented by $W_{Z}$

$$
W_{Z}=\left[w_{1}, w_{2}, \ldots, w_{N} 2\right] \quad \forall k=1, \ldots, t
$$

and a biped robot with $C O M$ at a given height $z_{c}$,

$$
x_{C O M} \in\left\{B_{r}\left[w_{1}\right], C^{2}\right\}
$$

where $B_{r}[\bullet]$ is the closed ball centered in $\bullet$ with diameter $S P_{t}$, which is the support polygon size at time $k$ subject to

$$
x_{Z M P}=x_{C O M}-\frac{z_{c} \ddot{x}_{C O M}}{g} .
$$

In order to implement the EPSAC control in the cart table model, first using $z_{c}=0.41 \mathrm{~m}$ and the state state representation,

$$
\begin{aligned}
& \dot{X}=A \cdot X+B \cdot U Z M P=C \cdot X+D \\
& A=\left[\begin{array}{lll}
0 & 1 & 0 \\
0 & 0 & 1 \\
0 & 0 & 0
\end{array}\right] \quad B=\left[\begin{array}{l}
0 \\
0 \\
1
\end{array}\right] C=\left[\begin{array}{lll}
1 & 0 & -z c / g
\end{array}\right] D=[0],
\end{aligned}
$$

then

$$
T F=\frac{-0.041794(s-4.892)(s+4.892)}{s^{3}}
$$

is the transfer function of the system used to get the model prediction. The step response is shown in Fig 5. It is observed that the non-minimum phase duration is $0.475 s$. The COM height of $z c=0.41 \mathrm{~m}$ is used to avoid knee singularity during walking. The sampling time used in the simulations is $T_{s}=5 \mathrm{~ms}$.
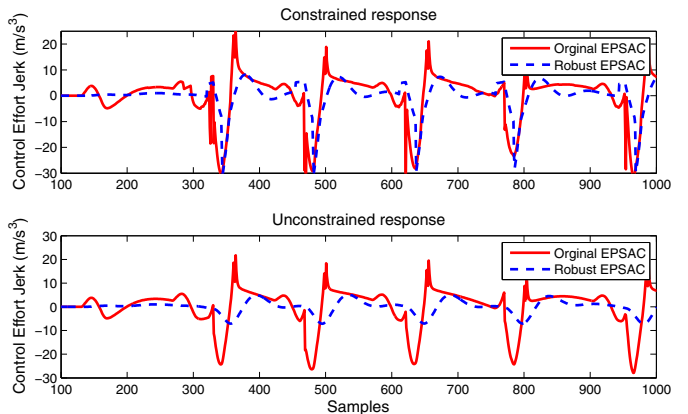

Fig. 6. Control effort for a fix trajectory $z_{c}=0.41 \mathrm{~m}$ $N_{u}=10$.

\subsection{ZMP Robustness Analysis}

A comparison study was done based on the same walking pattern to evaluate the performance of the system using the original EPSAC controller and the Robust EPSAC respectively. The control horizon was $N u=10$ and the prediction horizon was $N_{2}=400$ with a sampling time $T_{s}=5 \mathrm{~ms}$, resulting in a prediction of two seconds which is more than a single step for a biped gait. To generate a feasible gait, the required ZMP must be restricted within the support polygon through physical contacts. Therefore, to address the undershooting problem from instantaneously updating the ZMP reference inside the receding horizon, the acceleration constraints must be considered.

Since the real ZMP response should track the ZMP reference within the support polygon, a constrained zmp $\bar{x}_{p}$ should match a saturation such that

$$
x_{\text {foot }}-\Delta_{x}^{-}<\bar{x}_{p}<x_{\text {foot }}+\Delta_{x}^{+},
$$

is respected, where $\Delta_{x}^{+}, \Delta_{x}^{-}$is defined according to the size of the support polygon. The operator $\left(^{-}\right)$indicates the saturated state of the variable. These ZMP constraints, due to the support polygon, can be rewritten as acceleration constraints

$$
\overline{\ddot{x}}=\left\{\begin{array}{l}
\ddot{x}_{\text {min }}=\frac{x-\left(x_{\text {foot }}+\Delta_{x}^{+}\right)}{z_{c}} g, \\
\ddot{x}_{\text {max }}=\frac{x-\left(x_{\text {foot }}-\Delta_{x}^{-}\right)}{z_{c}} g,
\end{array}\right.
$$

The control responses for the constrained and unconstrained cases are shown in Fig. 6. In both cases, the control effort of the robust EPSAC is smooth and more continuous compared to the original EPSAC, reducing the high frequency components and the peaks of the input. In Fig. 7 the ZMP responses are presented. The figure shows that the original EPSAC has better tracking. On the other hand, our method generates a softer response but in the unconstrained case it does not follow the desired trajectory. However, once the constraints are imposed, the response tracks the trajectory with a behavior similar to the ideal case, allowing the implementation of the method. Fig. 8 shows the tracking error w.r.t the desired gait. According to the error analysis, the response of the original model remains inside the desired bound in all cases; the robust EPSAC violates the bound in the constrained case for a short period. This behavior is due to the difference between the optimization matrix $G$ once the method is applied and the nominal model used for the prediction. However, if a soft constraint is implemented, our implementation is feasible for specific applications. It is impor- 

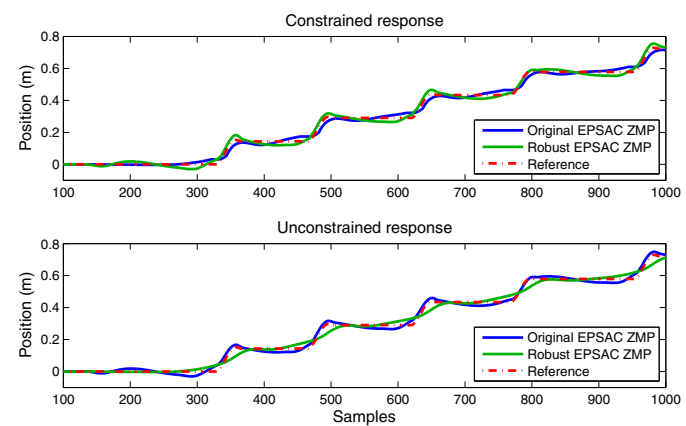

Fig. 7. ZMP response for a fix trajectory $N_{u}=10$.
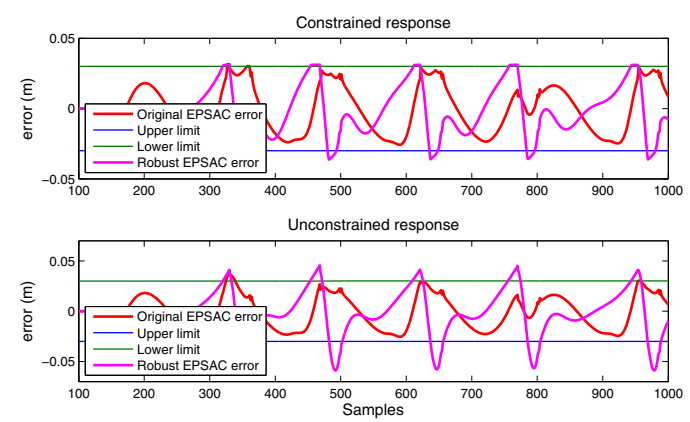

Fig. 8. ZMP response for a fixed trajectory $N_{u}=10$.

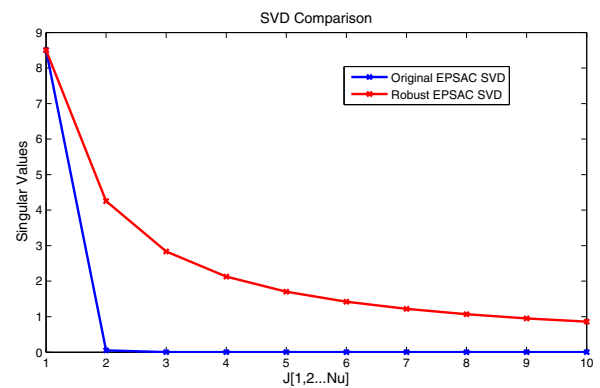

Fig. 9. SVD for the cart-table model $N_{u}=10$.

tant to remark that in this example, only the dynamically adjusted input constraint is used (16).

In order to test the robustness of the proposed method, a second set of simulations was carried out. The COM height of the model was set to $z_{c}=0.41 \mathrm{~m}$ and the desired gait trajectories were generated with an-online generator. The parameter $z_{c}$ in the simulated system was set to $z_{c}=0.38 \mathrm{~m}$, to analyze the behavior of the controller and its robustness, because in practice $z_{c}$ changes during a stable walk, (Murray et al. [1964]). In Fig. 9, the singular values for the original and robust EPSAC are compared. As it is shown, the dimensional space contains the solution of the original system. First, the original dynamics diverges without constraints even when the change on the COM height is less than $1 \mathrm{~cm}$. However, once the constraints are introduced, the performance of the system is not feasible since it oscillates around the desired trajectory as shown in Fig. 10. From a physical point of view, the response shows that the robot modifies the force distribution in the foot at a high frequency to track the trajectory, which causes vibrations and consequently the fall over. On the other hand, once we applied the robust EPSAC technique, the response was stable in both constrained and unconstrained cases as depicted in Fig. 11. In the first case, the system is able to track the trajectory properly according to the desired reference. In Fig. 12, the errors from the constrained and unconstrained cases are presented. The constrained response almost remains inside the desired bounds throughout the motion. The input dynamic has a soft constraint of $\pm 0.03 \mathrm{~m}$, since it must be within the size of the robot's feet.

\subsection{Simulation results}

The COmpliant HuMANoid Platform COMAN was modeled in the physics based simulation Open Dynamics Engine (ODE). The purpose of this simulation is to test the performance of the reactive gait generator before a future implementation on the real robot.

The COMAN robot is a whole body humanoid with 25 Degrees Of Freedom (DOF): 13 in the upper body, including neck, elbow, shoulders and waist and 6 DOF in each leg. The weight distribution and dimensions for COMAN can be found in (Tsagarakis et al. [2013]).

In the simulation, the real COM state feedback used to generate an online updated ZMP reference. The feet trajectories are consistently re-planned according to the ZMP

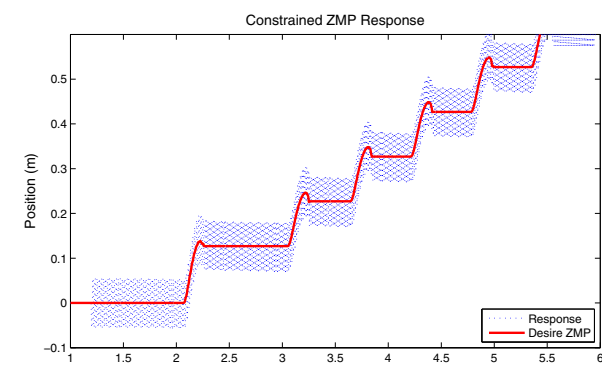

Fig. 10. ZMP responses when $z_{c}=0.38 \mathrm{~m}$.
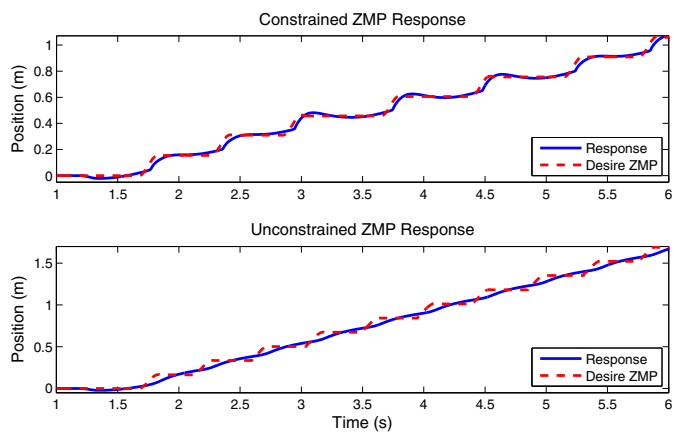

Fig. 11. ZMP responses when $z_{c}=0.38 \mathrm{~m}$.

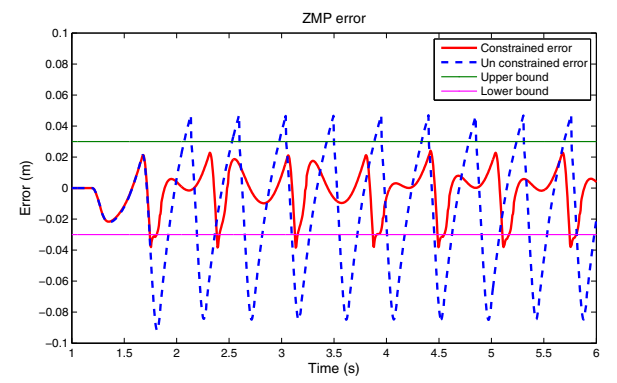

Fig. 12. ZMP responses when $z_{c}=0.38 \mathrm{~m}$. 


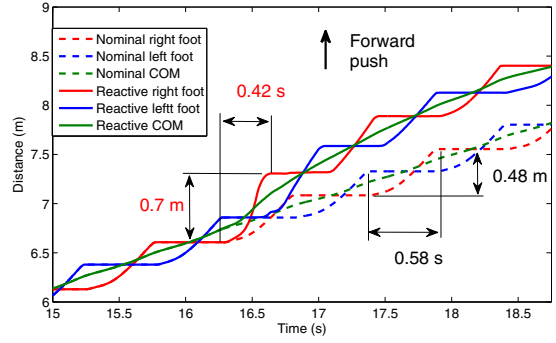

Fig. 13. Simulated response of COMAN humanoid.

reference as well. All these are done at every sampling time. The Robust EPSAC controller tracks the re-planned ZMP reference considering the acceleration constraints and generates an adaptive COM motion. The COM and feet trajectories are given to the inverse kinematics to obtain the joint trajectories for the joint position controllers. The feet were kept parallel to the ground. The selected gait for the presented example is: step length $S L=0.25 \mathrm{~m}$, step cycle $T s=0.6 \mathrm{~s}$, constant COM height $z_{c}=0.38 \mathrm{~m}$, sampling time $T s=5 \mathrm{~ms}$.

The disturbance response is shown in Fig.13. The disturbance was created by a flying object that hit the back of the robot with a horizontal speed of $11 \mathrm{~m} / \mathrm{s}$ and a mass of $0.5 \mathrm{~kg}$. As it is shown, the step length is adjusted in order to counterbalance the disturbance and maintain a stable gait. Once the disturbance is compensated, the robot recovers to the periodic gait.

\section{CONCLUSIONS}

In this contribution, a Robust Model Predictive Control has been proposed based on the analysis of the singular value decomposition in the Extended Prediction Self Adaptive Control approach to MPC. The method is essentially required for the case of long control horizons where numerical instability downgrades the control performance. First, the proposed method is tested in a well-conditioned but challenging system, an inverted pendulum, showing that the methodology improves the stability and robustness of the original EPSAC controller. Second, the robust constrained EPSAC controller is demonstrated to smoothly track a biped walking pattern using for a biped robot. It is shown that in this application a control horizon larger than one sample is required to achieve the desired performance, which is necessary to guarantee a feasible, stable and robust implementation of MPC. Finally, it is concluded that the proposed methodology based on SVD is well suited for the systems that require of a control horizon larger than one sample in the control Horizon $N_{u}$.

\section{REFERENCES}

A. Bagheri and B. Miripour-Fard. Implementation of the model predictive control for on-line trajectory planning of a walking robot subjected to external disturbances. In 2011 International Symposium in Innovations in Intelligent Systems and Applications (INISTA), pages 590-594, 2011.

R. De Keyser. Model Based Predictive Control, chapter Invited Chapter in UNESCO Encyclopaedia of Life Support Systems (EoLSS)., page 30 p. Article contribution
6.43.16.1, Eolss Publishers Co Ltd, Oxford, ISBN 09542 989 18-26-34 (www.eolss.net), 2003.

R. De Keyser and C.M. Ionescu. The disturbance model in model based predictive control. In Control Applications, 2003. CCA 2003. Proceedings of 2003 IEEE Conference on, volume 1, pages 446-451, 2003.

CPO Edmond Ayyappa. Normal Human Locomotion, Part 1: Basic Concepts and Terminology, volume 9. Journal of Prosthetics and Orthotics, 1997.

S. Kajita, F. Kanehiro, K. Kaneko, K. Fujiwara, K. Harada, K. Yokoi, and H. Hirukawa. Biped walking pattern generation by using preview control of zeromoment point. In 2003. Proceedings. ICRA '03. IEEE International Conference on, Robotics and Automation, volume 2, pages 1620-1626 vol.2, 2003.

Zhibin Li, NikosG. Tsagarakis, and DarwinG. Caldwell. Walking pattern generation for a humanoid robot with compliant joints. Autonomous Robots, 35(1):1-14, 2013. ISSN 0929-5593. doi: 10.1007/s10514-013-9330-7.

J.M. Maciejowski. Predictive Control: With Constraints. Pearson Education. Prentice Hall, 2002. ISBN 9780201398236.

Pat M. Murray, Bernard A. Drought, and Ross C. Kory. Walking Patterns of Normal Men. J Bone Joint Surg Am, 46(2):335-360, March 1964.

M. Parsa and M. Farrokhi. Robust nonlinear model predictive trajectory free control of biped robots based on nonlinear disturbance observer. In 2010 18th Iranian Conference on, Electrical Engineering (ICEE), pages 617-622, 2010.

O.J. Rojas and G.C. Goodwin. On the asymptotic properties of the hessian in discrete-time linear quadratic control. In American Control Conference, 2004. Proceedings of the 2004, volume 1, pages 239-244 vol.1, 2004.

Osvaldo J. Rojas, Graham C. Goodwin, Mara M. Sern, and Arie Feuer. An svd based strategy for receding horizon control of input constrained linear systems. International Journal of Robust and Nonlinear Control, 14(13-14):1207-1226, 2004. ISSN 1099-1239.

J.M.M. Sánchez and J. Rodellar. Adaptive Predictive Control: From the Concepts to Plant Optimization. PrenticeHall International Series in Systems and Control Engineering. Prentice Hall PTR, 1996. ISBN 9780135148617.

Nikos G. Tsagarakis, Stephen Morfey, Gustavo Medrano Cerda, Li Zhibin, and Darwin G. Caldwell. Compliant humanoid coman: Optimal joint stiffness tuning for modal frequency control. In Robotics and Automation (ICRA), 2013 IEEE International Conference on, pages 673-678, 2013.

Bram Vanderborght. Dynamic Stabilisation of the Biped Lucy Powered by Actuators with Control stiffness. Springer, 2010.

Miomir Vukobratovic and Davor Juricic. Contribution to the synthesis of biped gait. Biomedical Engineering, IEEE Transactions on, BME-16(1):1-6, 1969. ISSN 0018-9294.

Miomir Vukpbratovic and Branislav Boravac. Zeromoment point - thirty five years of its life. International Journal of Humanoid Robotics, 01(01):157-173, 2004.

L. Wang. Model Predictive Control System Design and Implementation Using MATLAB®. Advances in Industrial Control. Springer, 2010. ISBN 9781849968362. 